\title{
Designing and Launching Coronavirus Disease 19 Electronic Registry in Shiraz, Iran: A Brief Report
}

\author{
Ali Akbari $^{\circledR}$, Mohsen Moghadami $^{\circledR}{ }^{\circledR}$, Younes Ghasemi $^{\circledR}{ }^{\circledR}$, Mehrdad Sharifi $^{\circledR}$, Abdolrasoul Hemmati $^{5}$, \\ Abdolkhalegh Keshavarzi ${ }^{6}$, Fatemeh Javanmardi ${ }^{\circledR}{ }^{\circledR}$, Amir Emami ${ }^{*{ }^{*}}$ \\ ${ }^{1}$ Department of Anesthesiology, School of Medicine, Shiraz University of Medical Sciences, Shiraz, Iran. \\ ${ }^{2}$ Health Policy Research Center, Institute of Health, Shiraz University of Medical Sciences, Shiraz, Iran. \\ ${ }^{3}$ Department of Pharmaceutical Biotechnology, School of Pharmacy and Pharmaceutical Sciences Research Center, Shiraz \\ University of Medical Sciences, Shiraz, Iran. \\ ${ }^{4}$ Emergency Medicine Department, Faculty of Medicine, Shiraz University of Medical Sciences, Shiraz, Iran. \\ ${ }^{5}$ Department of Pharmacology, Shiraz University of Medical Sciences, Iran. \\ ${ }^{6}$ Surgery Department, General Surgery, Burn and Wound Healing Research Center, Shiraz University of Medical Sciences, \\ Iran. \\ ${ }^{7}$ Microbiology Department, Burn and Wound Healing Research Center, Shiraz University of Medical Sciences, Shiraz, Iran.
}

*Correspondence to Amir Emami,

Email: emami_a@susm.ac.ir emami.microbia@gmail.com

Received April 20, 2020 Accepted May 4, 2020 Published online June 30,2020

\begin{abstract}
Introduction: The emergence of severe acute respiratory syndrome coronavirus 2 (SARSCoV-2) has created a global concern for public health. Having sufficient data is the first step to understand the behavior of contagious diseases such as the COVID-19 pandemic. Although various studies have assessed COVID-19 features, there are many limitations about patients' characteristics, complications, and outcomes in different countries including Iran. The present study reported launching an electronic database for COVID-19 patients in Fars province, Iran. Method: A comprehensive web-based multicenter registry was designed and launched by Shiraz University of Medical Sciences in order to collect all information about COVID-19 hospitalized patients in Fars province, Iran.

Results: In this registry, patients' demographic characteristics, chest computed tomography scan findings, laboratory tests, complications during hospitalization, treatments, and disease course in Intensive Care units are recorded on a web-based electronic database. The virtual statues of the patient's family are evaluated by telephone calls, and the proceeding will be implemented for quarantine and hospitalization if required.

Conclusion: The registry is hoped to help all scientists to understand the current challenges and be prepared for possible future waves of the epidemic. Finally, this registry is a resource for all researchers who are interested in coronavirus and plays an important role in supporting the scientific community on the frontlines of combating the virus.

Keywords: COVID-19, Severe acute respiratory syndrome coronavirus 2, Registry, Iran
\end{abstract}

\section{Introduction}

According to previous reports from the World Health Organization (WHO), respiratory diseases have represented serious problems for public health over the last twenty years. In December 2019, several cases of pneumonia with unknown etiology were detected in Wuhan, China. Investigations showed that this illness was related to coronavirus family (Coronavaride) thus it was named COVID-19 by the WHO. ${ }^{1,2}$ This new coronavirus created a big challenge all over the world since it was very contagious and rapidly-spreading. By the emerging severe acute respiratory syndrome coronavirus 2 (SARS-CoV-2) as a respiratory disease, the outbreak was defined as a Public Health Emergency of International Concern by the WHO and became a pandemic. ${ }^{3,4}$ One of the most important problems related to this disease is the prolonged incubation period which makes the prediction complicated. Based on different reports, the incubation period seems to be within 5-14 days following exposures thus asymptomatic cases impress all predictions and decisions. ${ }^{5}$ By increasing the number of patients all

(c) 2020 The Author(s); Published by Zabol University of Medical Sciences. This is an open-access article distributed under the terms of the Creative Commons Attribution License (http://creativecommons.org/licenses/by/4.0), which permits unrestricted use, distribution, and reproduction in any medium, provided the original work is properly cited. 
over the world, the most effective initial managements are infection control measures which are defined as social distancing and immediate isolation of infected patients. ${ }^{6}$

Exact information about infected individuals, symptoms, and specific characteristics is required in order to clarify the uncertainties and ambiguities of this respiratory syndrome. Thus, a comprehensive database about patients with COVID-19 help specialists and health policymakers to reduce the transmission rate and mortality. ${ }^{7}$ Despite health authorities' efforts, Iran was not an exception to this outbreak. So far (8 April 2020), there has been no specific treatment for COVID-19. To have better management in the prevention, treatment, and diagnosis of patients, it is of utmost importance to conduct high-quality research on COVID-19. Hence, the present study aimed to establish an electronic registration system for suspected and confirmed individuals with COVID-19 at Shiraz University of Medical Sciences, Iran.

\section{Materials and Methods tudy Design and Settings}

From the beginning of the COVID-19 outbreak in Fars province, a comprehensive form was designed based on the recommended WHO checklist. ${ }^{8}$ All suspected and confirmed individuals with COVID-19 will be followed during hospitalization and one month afterward. Moreover, family members are screened and hospitalized if required. The current registry is designed in compliance with standard STORBE (Strengthening the Reporting of Observational Studies in Epidemiology) guidelines, along with the Declaration of Helsinki principles. ${ }^{9,10}$ It should be noted that this registry is approved by Shiraz University of Medical Sciences under the ethical code of IR.SUMS. REC.1399.022

\section{Inclusion and Exclusion Criteria}

Initial information was recorded for individuals with COVID-19 symptoms (i.e., fever, cough, and dyspnea) who referred to different centers in Fars province, Iran. To confirm the disease, the chest computed tomography (CT) scan and real-time polymerase chain reaction (RTPCR) test were performed for all patients. On the other hand, individuals were excluded from the registry if they were not satisfied with information recording and the follow-up.

\section{Bias and Quality Assurance}

As it is known, SARS-CoV-2 is a new infection and the disease has an unknown nature thus treatment protocols and guidelines may change during the study. Hence, a committee consisting of specialists in all aspects of this multi-factorial disease is meticulously monitoring data collection procedures. Table 1 provides information about the quality control team and the involved specialists.
Table 1. Members of the COVID-19 Crisis Committee of Shiraz University of Medical Sciences

\begin{tabular}{ll}
\hline Name & Affiliation \\
\hline Akbari, Ali & Anesthesiologist/MD \\
Emami, Amir & Microbiologist/Ph.D. \\
Fadakar, Nima & Neurologist/MD \\
Falahati, Farshad & Treatment Management/MD \\
Ghasemi, Younes & Pharmaceutical Biotechnologist/MD \\
Hashemi Zadeh Fard Haghighi, Leila & Nurse/Bachelor \\
Heidari, Mohammad Reza & Pharmacology/ PhD \\
Javanmardi, Fatemeh & Biostatistician/M.Sc \\
Keshavarzi, Abdolkhalegh & Surgeon/MD \\
Kojuri, Javad & Cardiologist/MD \\
Lotfi, Mehrzad & Radiologist/MD \\
Mansouri, AliReza & Treatment Management/MD \\
Moghadami, Mohsen & Infectious Disease Specialist/MD \\
Rezaei, Tahereh & Nurse/M.Sc. \\
Sharifi, Mehrdad & Emergency Medicine Specialist/MD \\
Shirazi Yeganeh, Babak & Pathologist/MD \\
\hline Note. In an alphabetical order/all members are affiliated with Shiraz \\
\hline Univerity of Mediscal
\end{tabular}

University of Medical Sciences.

\section{Results}

\section{Measures and Outcomes}

After initial information gathering, chest CT scan findings, laboratory tests, and complications during hospitalization, treatments, and the intensive care unit course were recorded on a web-based electronic database (ardr.sums. ac.ir). Then, the virtual statues of the patient's family were evaluated by telephone calls, and the proceedings are implemented for quarantine and hospitalization if necessary. Figure 1 displays the diagnostic algorithm for people with respiratory symptoms. In addition, flow charts for assessing the COVID-19 risk in pregnant women and neonates are provided in Figures 2 and 3, respectively.

Further investigations are performed for inpatients during the hospital stay. Table 2 presents related investigations for COVID-19 patients.

\section{Discussion}

Since COVID-19 spread across the world, public health has been confronted by a serious crisis. According to clinical manifestations, the severity of the symptoms of this respiratory infection ranges from a common cold-like illness to a severe viral pneumonia that may cause death. ${ }^{12}$ The greatest risk of COVID-19 is rapid transmission communities. To date (8 April 2020), COVID-19 has affected 209 countries and territories with 1,491,785 confirmed cases. In Iran, total cases and total death are 64586, and 3993, respectively (8 April 2020). ${ }^{13}$ According to different works of literature, most victims are susceptible individuals thus special attention is needed for elderly people, medical staff, immunosuppressed patients, pregnant women, and those with other underlying ${\text { diseases. }{ }^{14}}^{14}$ 


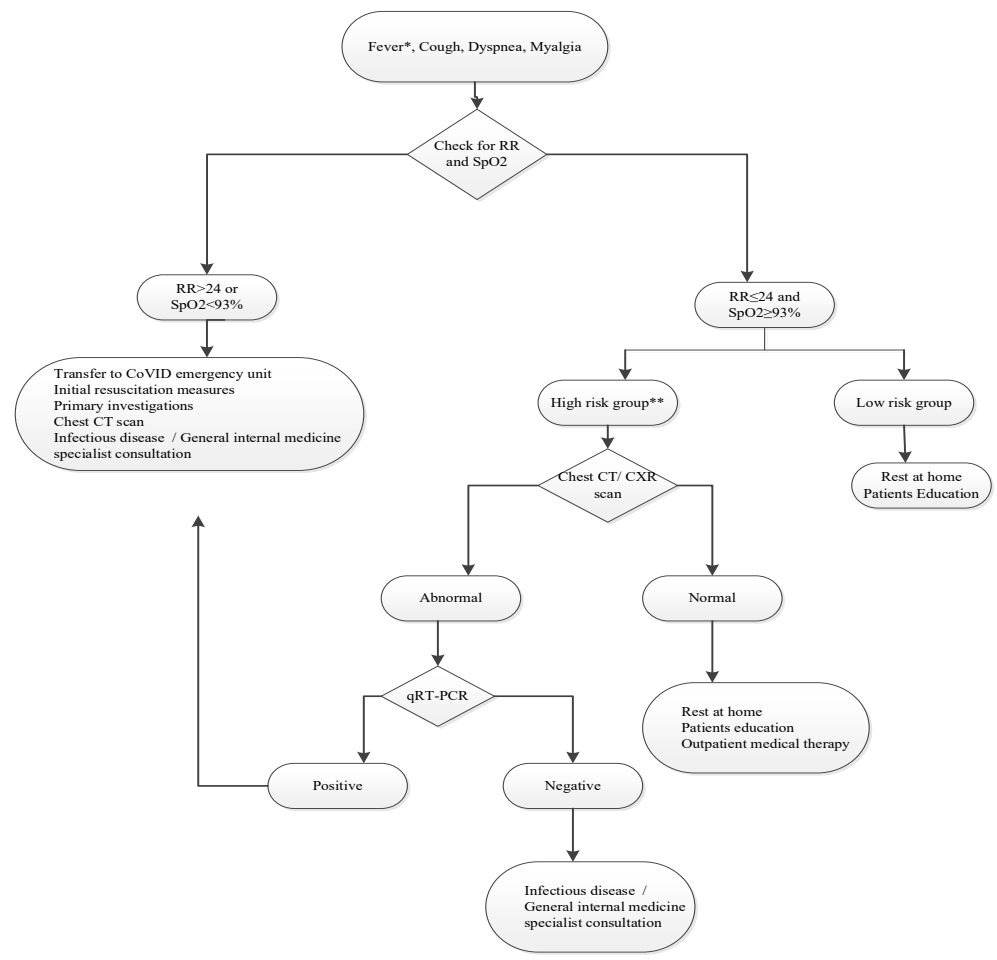

Figure 1. Algorithm of Patient Care for Individuals Presented With Respiratory Symptoms (Iran Ministry of Health and Medical Education, http://ird.behdasht.gov.ir)

Note. ${ }^{*}$ Fever: Temperature $\geq 37.3^{\circ} \mathrm{C}$ and $\geq 37.7^{\circ} \mathrm{C}$ in the morning and evening, respectively; ${ }^{* * H i g h-r i s k}$ groups: Immunocompromised patients including those with malignancy, organ transplant, HIV, cardiovascular diseases, diabetes, cirrhosis, and aged more than 50 .

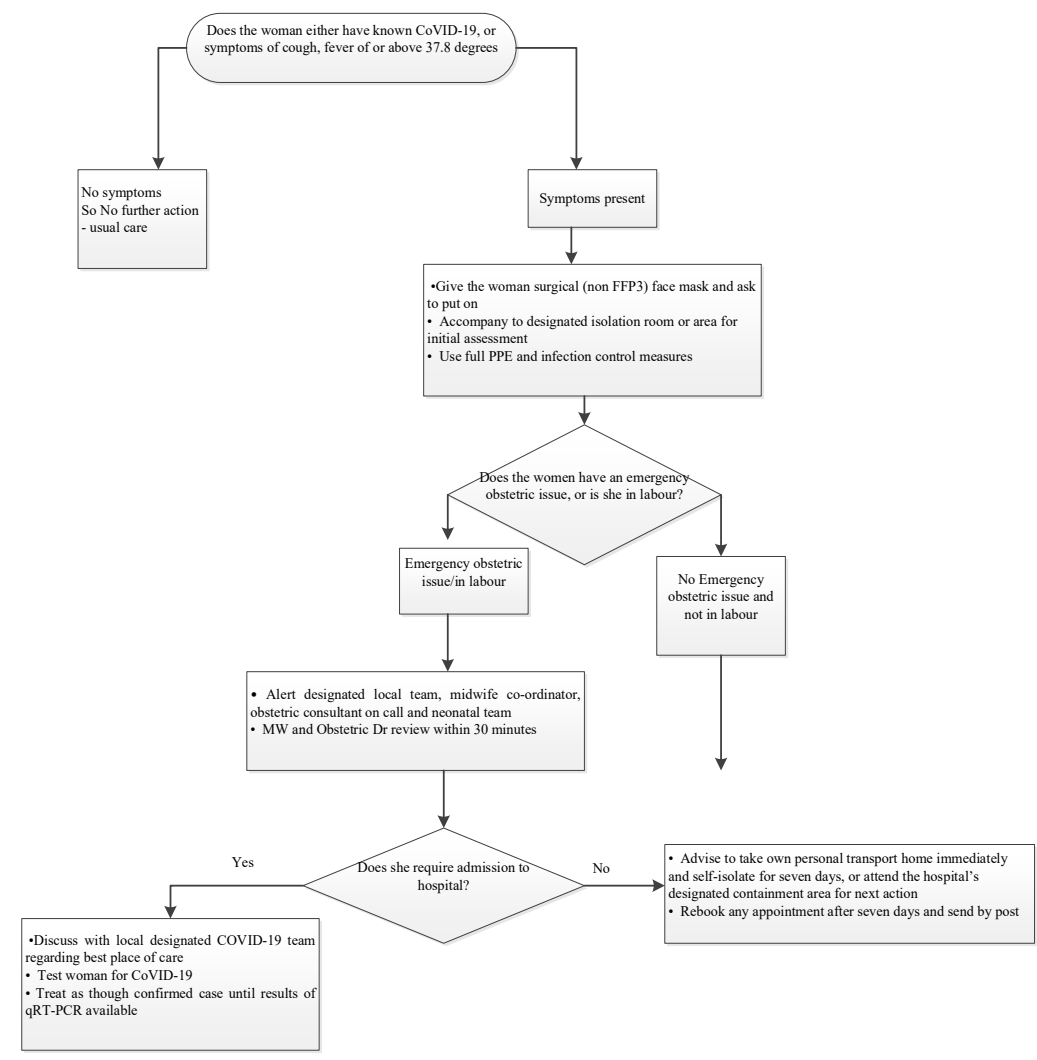

Figure 2. Flow Chart for Assessing COVID19- Risk in Maternity Unit Attendees Note. Adapted from Ortiz et al. ${ }^{11}$ PPE: Personal equipment protective; MW: Midwife. 


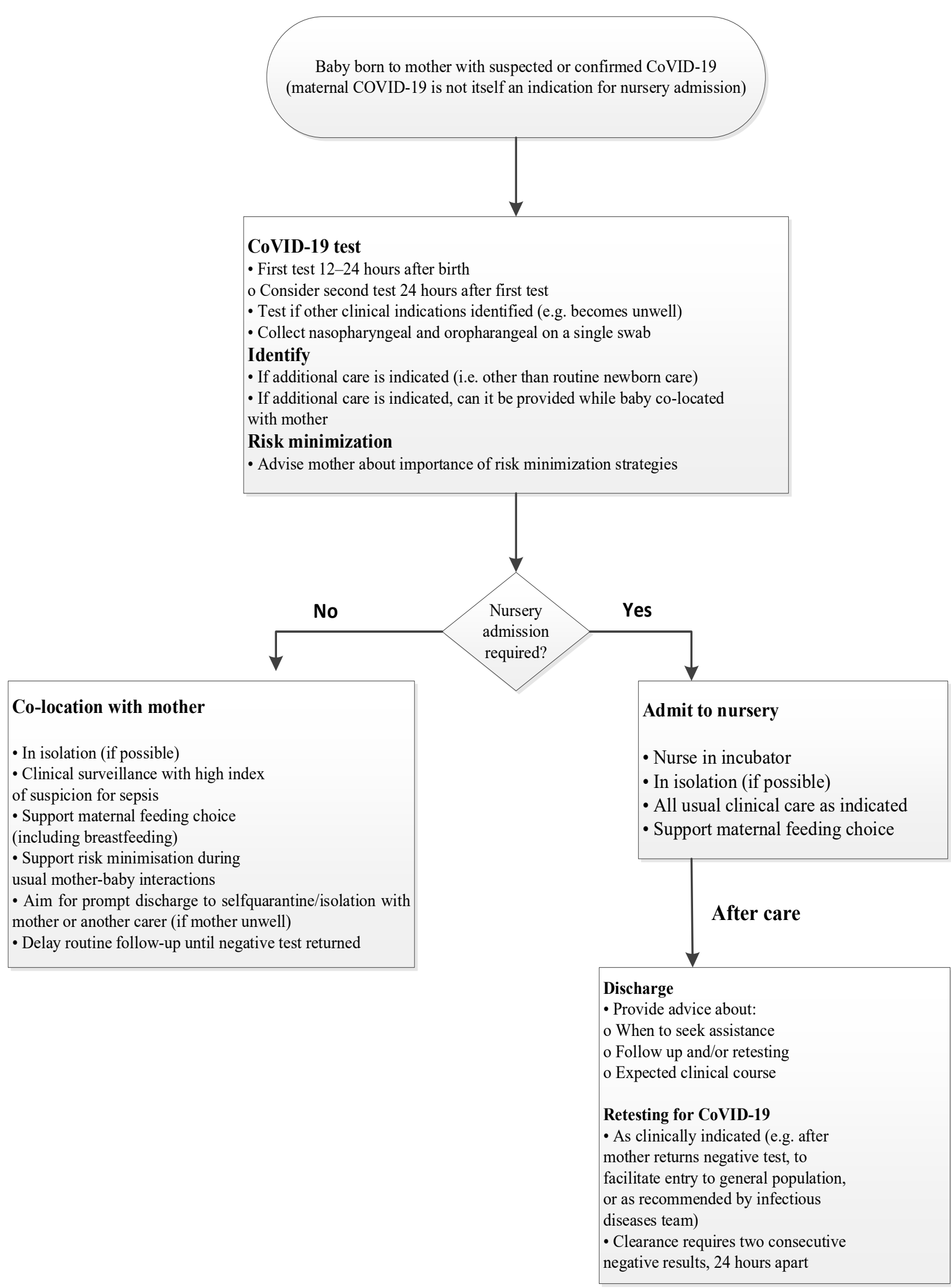

Figure 3. Neonates of Suspected or Confirmed COVID-19 Mothers.

Note. Adapted from https://www.health.qld.gov.au/qcg. Risk minimization strategies: 1. Hand hygiene before and after contact; 2. Cough or sneeze into elbow; 3. Face mask during baby care; 4 . Visitor restrictions; 5 . Cleaning/sterilizing equipment and surfaces. 
Table 2. Complementary Investigations in Patients With COVID-19

\begin{tabular}{|c|c|}
\hline \multicolumn{2}{|l|}{ All Patients } \\
\hline \multirow{9}{*}{ Laboratory tests } & Complete blood count with differential \\
\hline & CRP \\
\hline & Serum creatinine \\
\hline & Liver function tests \\
\hline & Ferritin \\
\hline & Blood group \\
\hline & Myoglobin \\
\hline & Creatinine kinase \\
\hline & $\mathrm{Na}, \mathrm{K}, \mathrm{Mg}$, and vitamin $\mathrm{D}$ \\
\hline \multirow{5}{*}{ Chest CT scan } & Density (i.e., ground-glass opacity, consolidative opacity, and mixed) \\
\hline & Internal characteristics of the lesion (i.e., crazy-paving and reversed halo sign) \\
\hline & Axial location and lobar location \\
\hline & Shape (i.e., patchy, round-nodular, and confluent) \\
\hline & $\begin{array}{l}\text { Other findings (i.e., tree-in-bud opacities, predominantly nodular opacities, predominantly reticular opacities, cavitation, mediastina } \\
\text { lymphadenopathy, and pleural effusion) }\end{array}$ \\
\hline \multicolumn{2}{|c|}{ ICU admitted patients } \\
\hline Laboratory tests & $\begin{array}{l}\text { PT, PTT, INR } \\
\text { D-dimer }\end{array}$ \\
\hline ICU course sheet & $\begin{array}{l}\text { APACHE score, SOFA score } \\
\text { Types of oxygen therapy (i.e., reservoir bag, CPAP, NIV, and intubation) } \\
\text { Oxygen statues (i.e., } \mathrm{SPO}_{2}, \mathrm{PAO}_{2} / \mathrm{FiO}_{2} \text {, and } \mathrm{PAO}_{2} \text { during and after intubation) } \\
\text { Lung dynamics (compliance and resistance) } \\
\text { Advanced hemodynamic parameters with USCOM } \\
\text { Complication and outcomes }\end{array}$ \\
\hline \multicolumn{2}{|l|}{ If sepsis suspected } \\
\hline \multirow{3}{*}{ Laboratory test } & Arterial blood gas \\
\hline & Procalcitonin \\
\hline & Blood culture \\
\hline
\end{tabular}

Note. CRP: C-reactive protein; Na: Sodium; K: Potassium; Mg: Magnesium; CT scan: Computed tomography scan; ICU: Intensive Care units; PT: Prothrombin time; PTT: Partial thromboplastin time; INR: International normalized ratio; APACHE: Acute physiology and chronic health evaluation; SOFA: Sequential organ failure assessment; CPAP: Continuous positive airway pressure; NIV: Non-invasive ventilation; USCOM: Ultrasonic cardiac output monitor; SPO $_{2}$ : Peripheral oxygen saturation; $\mathrm{PAO}_{2} / \mathrm{FiO}_{2}$ : Fraction of inspired oxygen/Fraction of inspiratory oxygen.

Although this new virus is from the same family of SARS-CoV, various ambiguous points exist, and prior recommendations are insufficient to confine the disease. To block the routes of transmission, epidemiological changes should be monitored carefully and further studies are essential, especially since no one is assured of SARS-CoV-2 immunity. ${ }^{15}$ Accordingly, a comprehensive database is helpful to support medical researchers and healthcare professionals in their efforts regarding better understanding and combating the disease associated with this novel coronavirus. In this manuscript introduced our electronic web-based registry, which was designed and launched by Shiraz University of Medical Sciences. In this system, monitoring patients and frequently checking back for additional content could be implemented with detail. According to laboratory tests, we could also find a cut-off to predict the disease in suspicious individuals. ${ }^{16} \mathrm{~A}$ similar registry has been set up to support rapid evidence synthesis in Europe and Tehran. ${ }^{17,18}$ It is hoped that this valuable database provides extensive knowledge about different aspects of this disease such as demographic characteristics, comorbidities, laboratory findings, risk factors, and the safety and efficacy of various treatments.

\section{Conclusion}

The current study provided a brief report about our electronic registry system for patients with COVID-19 in Fars province, Iran. For those researchers who are interested in the field and wish to follow the last updates on SARS-CoV-2 in particular, this registry helps them collect reliable and especially up-to-date information on this issue.

\section{Ethical Approval}

This study was approved by the ethical committee code of IR.SUMS.REC.1399.022.

\section{Conflict of Interest Disclosure}

The authors declare that they have no conflict of interests.

\section{Funding/Support}

This work was supported by the Vice-chancellor of Research and University Resources. 


\section{Authors' Contributions}

All authors drafted part of the manuscript and approved the final version.

\section{Acknowledgments}

All authors would like to thank the Vice-chancellor of Research for ethical approval, the Vice-chancellor of Management Development and University Resources for financial support, the Vice-chancellor of Treatment, the Vice-chancellor of Health, and the Vice-chancellor of Food and Drug for accessing the information and good cooperation.

\section{References}

1. Wang L, Wang Y, Ye D, Liu Q. Review of the 2019 novel coronavirus (SARS-CoV-2) based on current evidence. Int J Antimicrob Agents. 2020;55(6):105948. doi:10.1016/j. ijantimicag.2020.105948

2. Jiang F, Deng L, Zhang L, Cai Y, Cheung CW, Xia Z. Review of the clinical characteristics of coronavirus disease 2019 (COVID-19). J Gen Intern Med. 2020;35(5):1545-1549. doi:10.1007/s11606-020-05762-w

3. World Health Organization (WHO). Coronavirus Disease 2019 (COVID-19): Situation Report, 67. WHO; 2020.

4. Liu H, Wang LL, Zhao SJ, Kwak-Kim J, Mor G, Liao AH. Why are pregnant women susceptible to COVID-19? an immunological viewpoint. J Reprod Immunol. 2020;139:103122. doi:10.1016/j.jri.2020.103122

5. Lauer SA, Grantz KH, Bi Q, et al. The incubation period of coronavirus disease 2019 (COVID-19) from publicly reported confirmed cases: estimation and application. Ann Intern Med. 2020;172(9):577-582. doi:10.7326/m20-0504

6. Wu CI, Postema PG, Arbelo E, et al. SARS-CoV-2, COVID-19, and inherited arrhythmia syndromes. Heart Rhythm. 2020. doi:10.1016/j.hrthm.2020.03.024.

7. Guan WJ, Ni ZY, Hu Y, et al. Clinical characteristics of coronavirus disease 2019 in China. N Engl J Med. 2020;382(18):1708-1720. doi:10.1056/NEJMoa2002032

8. World Health Organization (WHO). Hosptal Readiness
Chekcklist for CoVID-19. WHO; 2020.

9. Vandenbroucke JP, von Elm E, Altman DG, et al. Strengthening the Reporting of Observational Studies in Epidemiology (STROBE): explanation and elaboration. Ann Intern Med. 2007;147(8):W163-194. doi:10.7326/00034819-147-8-200710160-00010-w1

10. World Medical Association Declaration of Helsinki: ethical principles for medical research involving human subjects. JAMA. 2013;310(20):2191-2194. doi:10.1001/ jama.2013.281053

11. Ortiz EI, Castañeda EH, De La Torre A. Coronavirus (COVID 19) infection in pregnancy. Colomb Med. 2020;51(2):e4271. doi:10.25100/cm.v51i2.4366

12. Şahin AR, Erdogan A, Ağaoğlu PM, et al. 2019 novel coronavirus (COVID-19) outbreak: a review of the current literature. Eurasian J Med Oncol. 2020;4(1):1-7. doi:10.14744/ejmo.2020.12220

13. Lin CY, Cheng CH, Lu PL, et al. Active surveillance for suspected COVID-19 cases in inpatients with information technology. J Hosp Infect. 2020;105(2):197-199. doi:10.1016/j.jhin.2020.03.027

14. Emami A, Javanmardi F, Pirbonyeh N, Akbari A. Prevalence of underlying diseases in hospitalized patients with COVID-19: a systematic review and meta-analysis. Arch Acad Emerg Med. 2020;8(1):e35.

15. Zhou P, Yang XL, Wang XG, et al. A pneumonia outbreak associated with a new coronavirus of probable bat origin. Nature. 2020;579(7798):270-273. doi:10.1038/s41586-0202012-7

16. Zheng $\mathrm{Y}$, Zhang $\mathrm{Y}$, Chi H, et al. The hemocyte counts as a potential biomarker for predicting disease progression in COVID-19: a retrospective study. Clin Chem Lab Med. 2020;58(7):1106-1115. doi:10.1515/cclm-2020-0377

17. Eular CoVID-19 database. https://www.eular.org/eular covid19_database.cfm.

18. Talebpour M, Hadadi A, Oraii A, Ashraf H. Rationale and design of a registry in a referral and educational medical center in Tehran, Iran: Sina Hospital Covid-19 Registry (SHCo-19R). Advanced Journal of Emergency Medicine. 2020;4(2S). doi:10.22114/ajem.v4i2s.361 